\title{
The Comparative Study on Smoke Control Effect of Longitudinal Ventilation and Ceiling Screen in Complex Tunnels
}

\author{
Zhi Sheng WANG ${ }^{1, a}$, Wan Fu LIU1 ${ }^{1}$, ZhaoPeng $\mathrm{NI}^{2}$ and Shi Chang LU2 \\ ${ }^{1}$ School of Mechanical Engineering, Tianjin University of Commerce, Tianjin, China \\ 2 Tianjin Fire Reasearch Institute of MPS, Tianjin,China \\ aemail:wzs1012@yeah.net
}

\begin{abstract}
Keywords:smoke control,longitudinal ventilation, ceiling screen
Abstract. With the development of China's transportation industry and the construction of traffic tunnels, tunnel disaster prevention has become an increasingly important research topic. In this paper, we compared the two kinds of smoke prevention modes in the vertical tunnel with the vertical ventilation and the vertical wall, and the results show that the vertical ventilation and smoke protection method can effectively control the flue gas in the tunnel without fire, escape. The smoke-proof mode of the smoke-laden wall can only slow the spread rate of flue gas, and its effect is poor compared with the longitudinal ventilation mode.
\end{abstract}

\section{Introduction}

According to the results of the fire survey, the real cause of the death of a large number of people is the toxic smoke generated in the fire [1]. This is mainly that the accumulation of flue gas in the tunnel reduces spatial visibility, which is easy to cause psychological panic and bring obstacles to the evacuation of personnel. So reasonable implementation of tunnel ventilation and smoke prevention measures can effectively control the spread of smoke to ensure that safe personnel evacuation in fire.

At present, longitudinal ventilation and horizontal smoke evacuation and other methods are mainly adopted for the domestic control of the smoke inside the tunnel, and a variety of ventilation and smoke evacuation methods are studied by many scholars [2]. The ceiling screen is a commonly used method for smoke control in tunnel fire [3,4,5]. The Sichuan Fire Research Institute of Ministry of Public Security has studied the application of ceiling screen. The results show that, in the building fire and for a certain period of time after the fire, ceiling screen can prevent the spread of large smoke out of the smoke prevention area [6]. Through the entity experiment, the comparisons have been made for the vertical ventilation and ceiling screen in working condition of the same fire load, which provides the basis for the reasonable prevention and control of smoke in the complicated highway tunnel project.

\section{The establishment of tunnel fire model}

\section{Design of experimental working conditions}

In this experiment, a city highway tunnel is taken as the reference object; reduced scale relationship is established according to $1: 3$ in the width and height of tunnel;the cross-sectional size of the main tunnel of reduced scale is $4.35 \mathrm{~m} \times 2 \mathrm{~m}$ (width $\times$ high); the cross-sectional size of branch tunnel model is $4.15 \mathrm{~m} \times 1.73 \mathrm{~m}$ (width $\times$ high). Since the intersection curve of the highway tunnel is smooth, the experiment sets arc whose curvature radius is $1.4 \mathrm{~m}$ in the main tunnel and branch tunnel sets arc whose curvature radius is $0.7 \mathrm{~m}$ in the intersection of the branch tunnel. The test bed model is shown in Figure 1. The tunnel which the source of the fire is located in adopts semi-horizontal smoke evacuation way, whose distance of the exhaust port is $10 \mathrm{~m}$ and smoke exhaust rate is $10.27 \mathrm{~m}^{3} / \mathrm{s}$. The blue dotted line is the flue. 


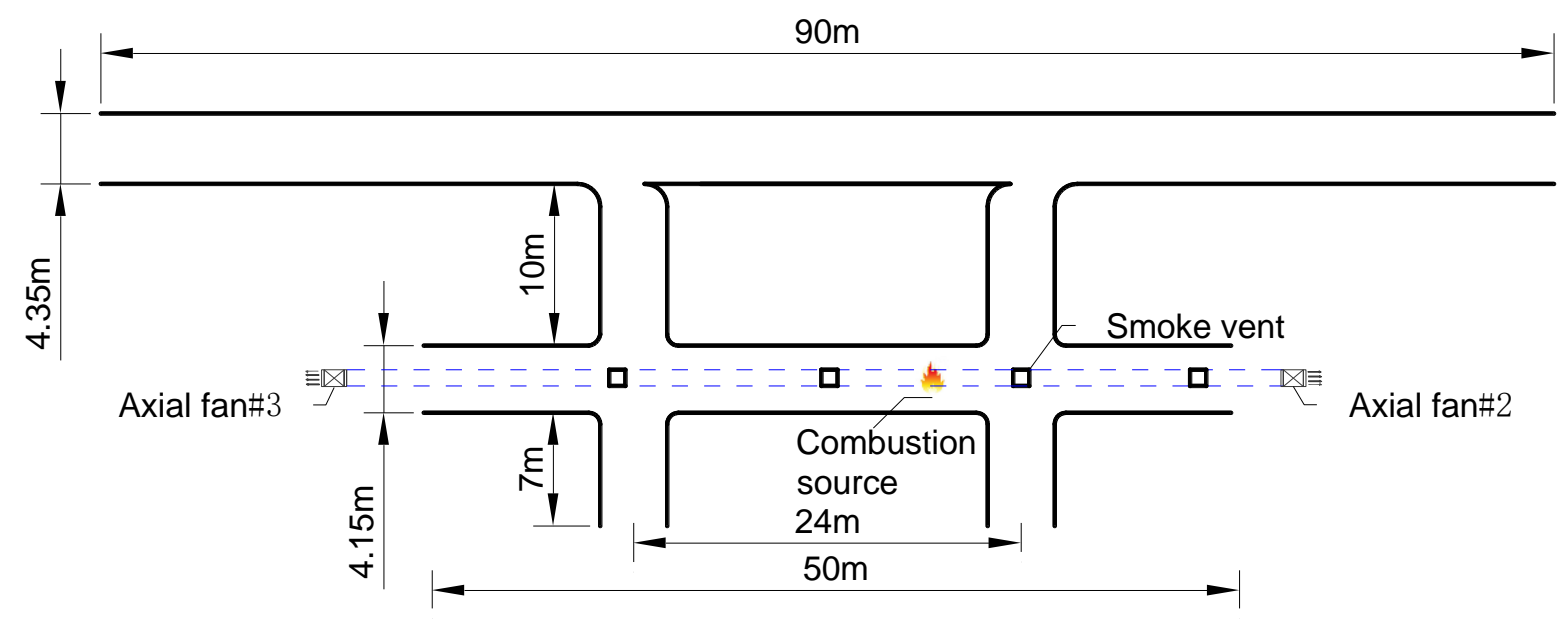

Fig. 1 Sketch map of experiment platform

The setting of fire scene

This topic sets the fire source in the tunnels of branch road, and the specific location is shown in Figure 1. When the fire source position is same, different smoke prevention and evacuation models are adopted to observe the impact of smoke on the main tunnel.

This experiment is divided into two kinds of working conditions, one is to set the axial fan to carry out the longitudinal ventilation for the entrance of main tunnel, and the other is to set ceiling screen for the main tunnel. The diagrams are shown in figures 2 and 3. T stands for thermocouples, and $\mathrm{CM}$ stands for cameras. The wind speed of the longitudinal ventilation is chosen as the critical velocity of the upstream branch slip tunnel, according to the formula [7]

$$
\begin{aligned}
& V_{c}=k_{g} k\left(\frac{g H Q}{\rho_{\infty} c_{p} A T_{f}}\right)^{1 / 3} \\
& T_{f}=\frac{Q}{\rho_{\infty} c_{p} A V_{c}}+T_{\infty}
\end{aligned}
$$

From this formula, $V_{c}$ is the critical wind velocity, $k_{g}$ is the slope correction factor, $\mathrm{g}$ is the gravitational acceleration, $H$ is the tunnel section height, $Q$ is the heat release rate, $\rho_{\infty}$ is the air density in tunnel, $c_{p}$ is the specific heat capacity of air at constant pressure, $A$ is the tunnel ventilation section area, $T_{f}$ is the hot gas temperaturethe, $T_{\infty}$ is the ambient temperature. By calculation, the critical wind speed is calculated to be $2.5 \mathrm{~m}$. Ying Zhigang has studied the performance of the air curtain and the ceiling screen to block the smoke, and obtained that the ceiling screen has more effective control of the smoke with the increase of the height. Therefore, in this subject, the ceiling screen is as high as the tunnel [8]. 


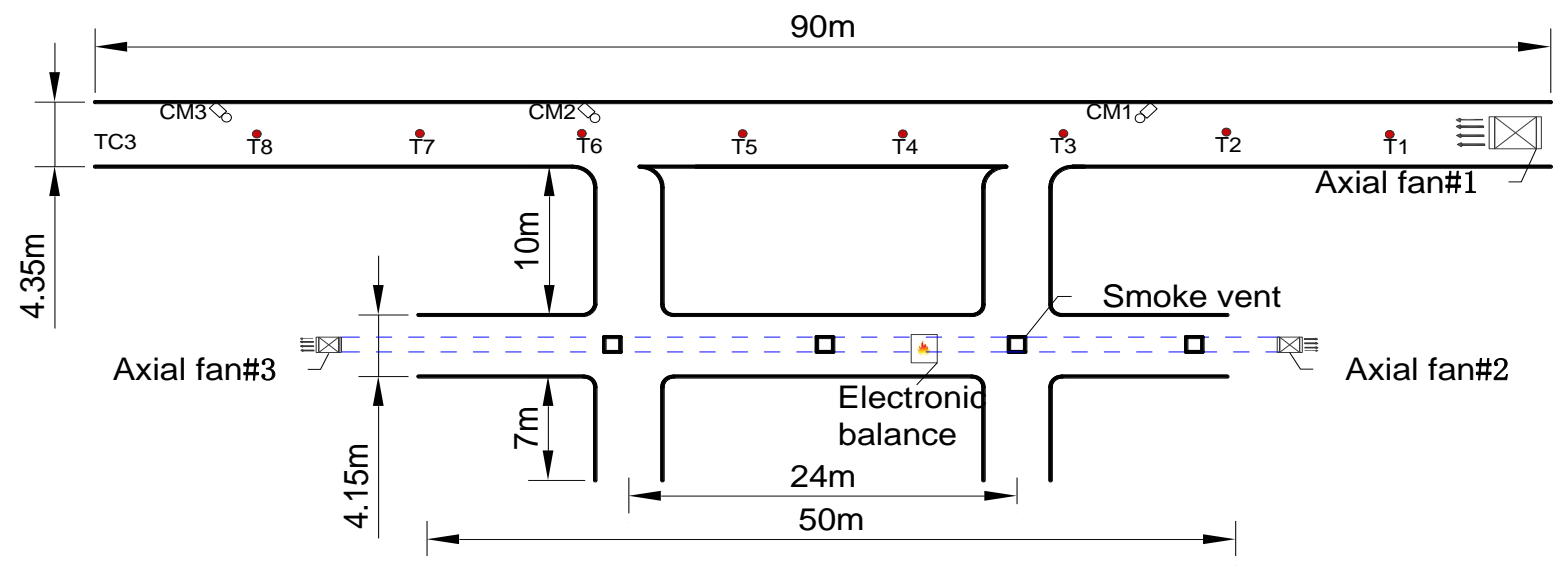

Fig. 2 Sketch map of longitudinal ventilation mode

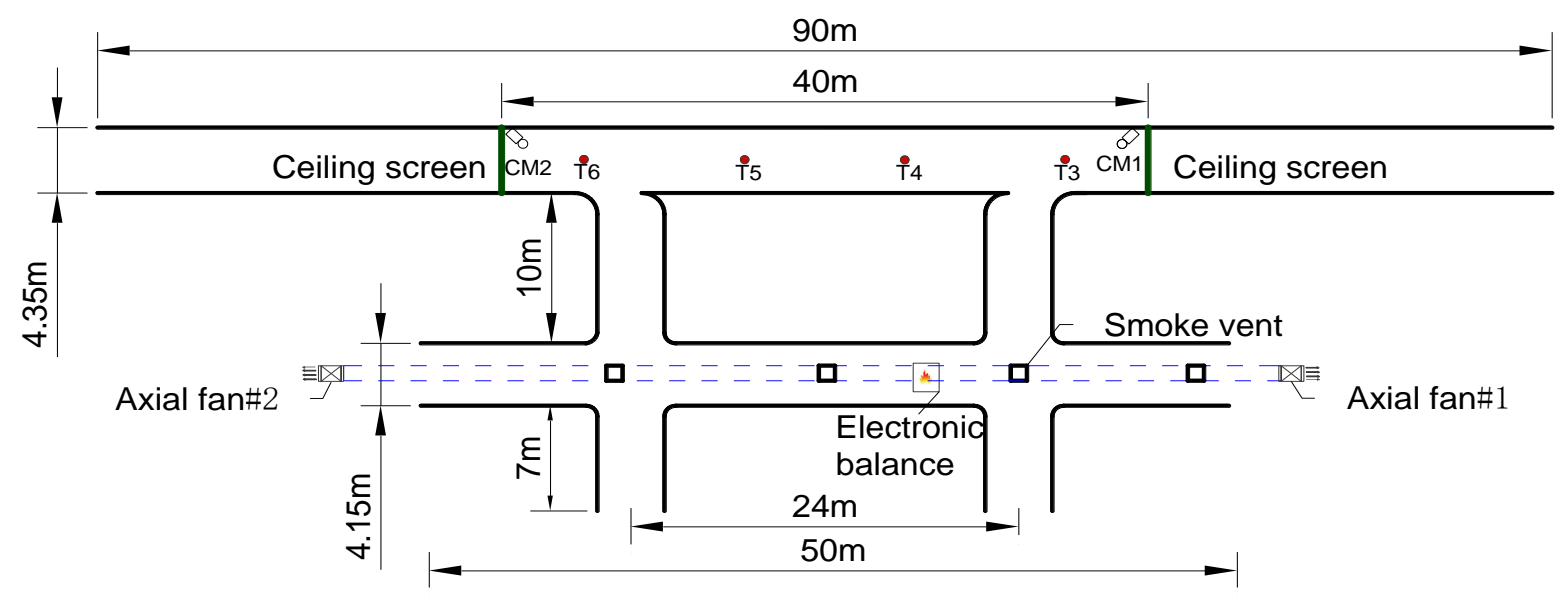

Fig. 3 Sketch map of ceiling screen mode

The setting of fire source

In this experiment, the prototype fire scale is $30 \mathrm{MW}$, and the fire load the experiment needs is $1.93 \mathrm{MW}$ according to the Froude criterion [9]. Weight loss method is used to calculate the heat release rate.

$\frac{Q_{m}}{Q_{n}}=\left(\frac{L_{m}}{L_{n}}\right)^{5 / 2}=1: 15.576$

(3) 


\section{Analysis of temperature change results}

The temperature variation of the longitudinal ventilation mode

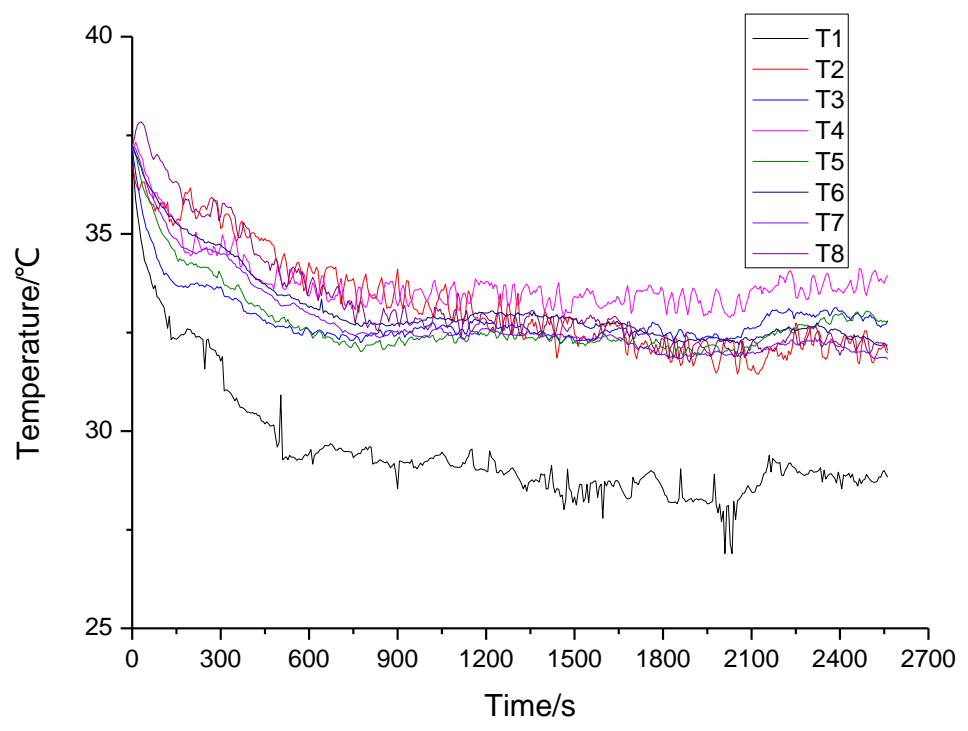

Fig. 4 The temperature variation of the longitudinal ventilation mode

It can be seen from the variation curve of the temperature in the main tunnel, the actual temperature at each temperature measurement point in the tunnel gradually decreases after the start of the experiment and remains constant after the 730s.It is concluded that since the temperature in the tunnel is higher than room temperature,after the axial fan is opened, the hot air in the tunnel is discharged from the tunnel and the new air is added, which makes the temperature decreased. Under normal circumstances the smoke temperature is higher than room temperature, but there is no temperature rise phenomenon in the main tunnel temperature, which can be obtained that no or only a small amount of smoke spread to the main tunnel.

Through the temperature analysis, it can be seen that the temperature in the tunnel can be effectively reduced by adopting the smoke evacuation mode of longitudinal ventilation in the tunnel without fire. At the same time, because the smoke temperature is generally higher than room temperature, but in the experimental process there is no phenomenon of the increase around the thermocouple temperature, which can be obtained that this mode can effectively prevent the spread of smoke into the tunnel.

The temperature variation of the ceiling screen

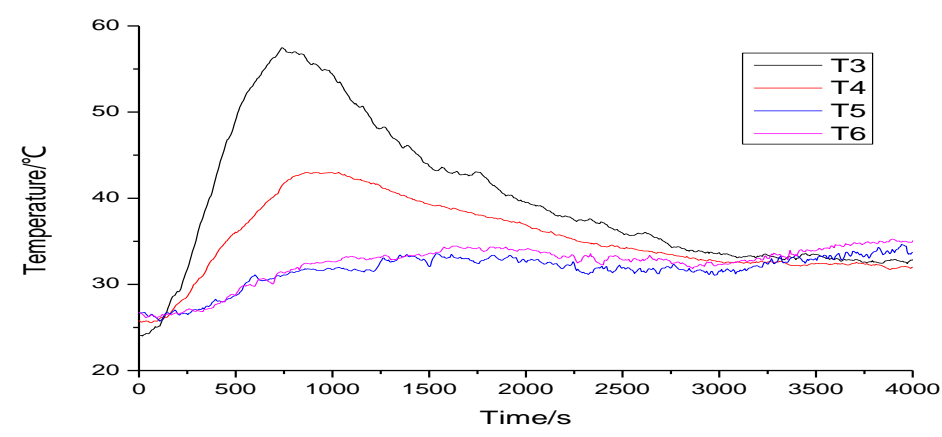

Fig. 5 The temperature variation of the ceiling screen

In the main tunnel, because the program needs to do the block, the thermocouple in the experimental area is reduced to four. From the temperature curve of main tunnel it can be seen that, after the commence of the experiment, there is a phenomenon of temperature rise at No.3 thermocouples firstly, then No. 4 thermocouple begins to rise in temperature in the 85th second, and 
No.5 and 6 thermocouple shows a significant rise of temperature in the 304th second. Through the comparison of the peak temperature it can be obtained, the rising speed of No.3 thermocouple is faster, and its peak temperature is higher, the peak appears in the first 739th seconds, the peak temperature is $57^{\circ} \mathrm{C}$. Then the No. 4 thermocouple from first $785 \mathrm{~s}$ to $1130 \mathrm{~s}$ reaches $42^{\circ} \mathrm{C}$, and its temperature rise rate is slower than the temperature of the No.3 thermocouple temperature. No.5 and 6 thermocouple rise more slowly, and the peak temperature is low, the measured values of them in the experiment is nearly same, there is a significant decline in two thermocouple after the temperature rises. No. 3 and 4 thermocouple decline rapidly after the peak temperature and the decline speed of No.3 is faster than No. 4. At the end of the experiment, the temperature of the four thermocouples are all about $33{ }^{\circ} \mathrm{C}$, which is higher than the temperature at the beginning of the experiment.

Through the analysis, it can be seen that the thermocouple of the main tunnel has obvious temperature change, the temperature rising trend is obvious and the speed is very fast, which indicates that the smoke flows into the main tunnel, and the entry speed is faster. Through the comparison of thermocouple position, it shows that closer the distance from the fire source, more obviously the thermocouple temperature rise.

\section{Experimental image analysis}

The analysis of mode image of the longitudinal ventilation

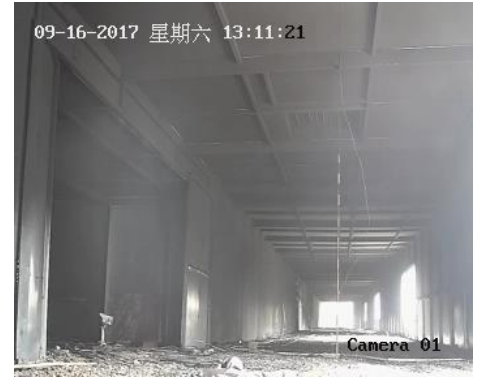

(a)

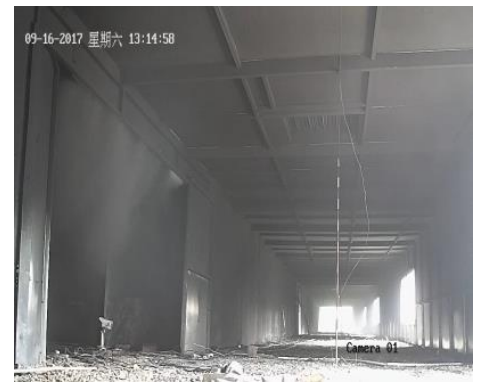

(d)

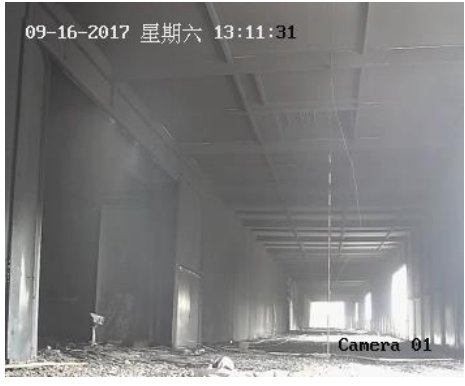

(b)

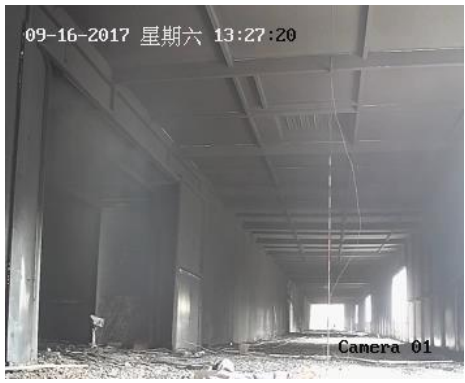

(e)

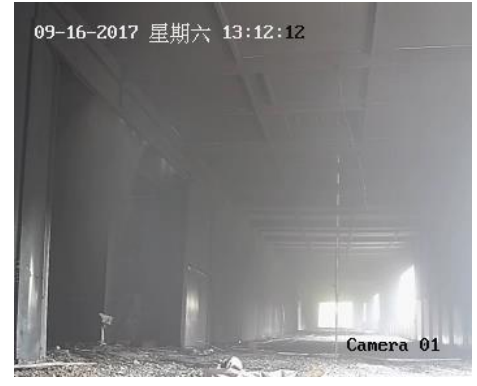

(c)

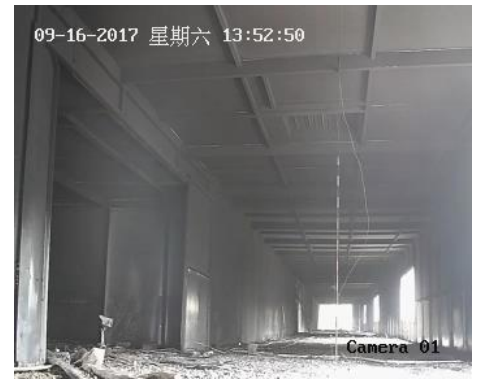

(f)

Fig. 6 The mode image of the longitudinal ventilation

Through the observation of the intersection of the branch road and the main tunnel, 141th second after the source ignited, the smoke is firstly observed, and part of the smoke spread to the main tunnel, smoke is observed firstly at the entrance of the loop, after that smoke gradually increases and the increasing speed is very quick, phenomenon of smoke and gas stratification can be observed, in the first 192th second the main tunnel visibility reaches minimum, at this point the thickness of smoke layer in the branch ways reaches $0.8 \mathrm{~m}$, then the smoke entering the main tunnel gradually reduces, visibility gradually increases, in the first 348th secondthere is almost no smoke spreading into the main tunnel, but at this time the thickness of the smoke layer of the branch way is still maintained at $0.8 \mathrm{~m}$, and the duration of its period is very long, in 1100th second the smoke gradually reduces, until the experiment ends, the flue gas decrease.

It can be seen that when there is a fire in a tunnel and the smoke model of the vertical 
ventilation is carried out at the adjacent tunnel, the wind speed at the tunnel intersection reaches the critical wind speed, which will effectively prevent smoke spread to no fire tunnel. In addition, when using the model, the tunnel is still connected state, which is conducive to personnel escape.

\section{The analysis of mode image of the ceiling screen}

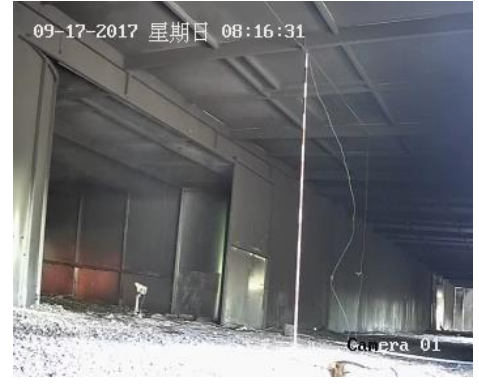

(a)

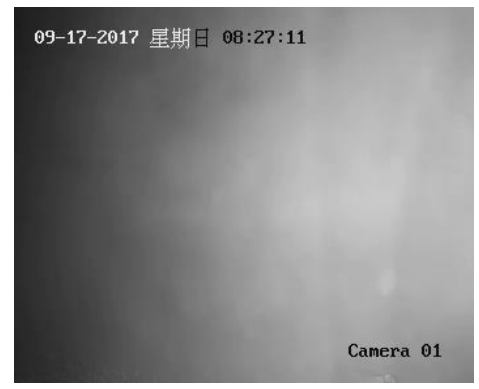

(d)

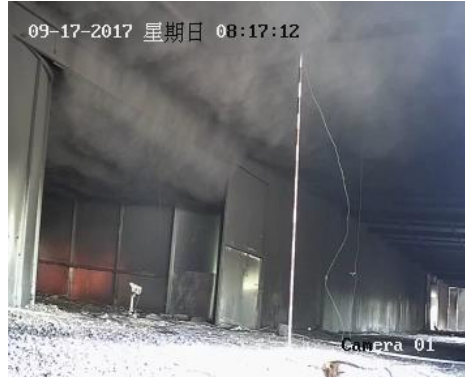

(b)

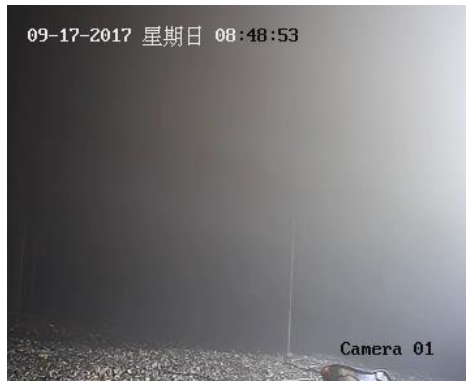

(e)

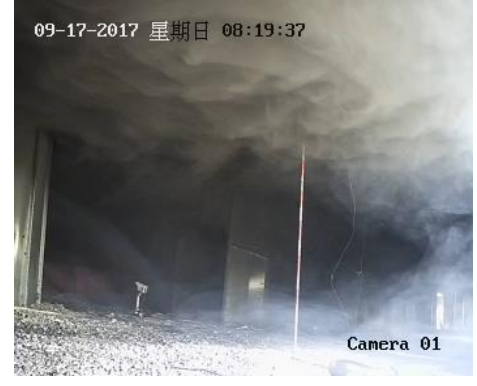

(c)

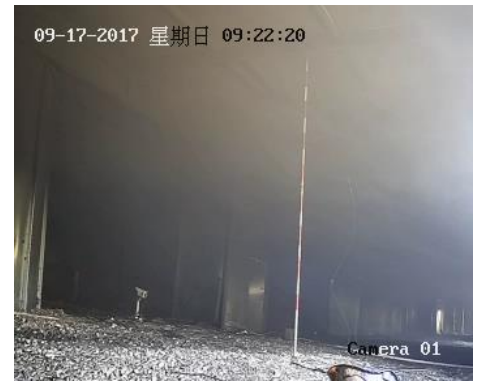

(f)

Fig. 7 The analysis of mode image of the ceiling screen

Through the observation of the intersection of the branch road and the main tunnel, 83th second since the source is ignited the smoke is firstly observed, smoke gradually spreads to the main tunnel, after that the smoke gradually increases and the speed increases quickly, at 120th second, from the image the obvious smoke and gas stratification phenomenon can be observed, in 270th second the thickness of the smoke layer in the main tunnel reaches $0.8 \mathrm{~m}$, the thickness of the smoke layer is gradually increasing, until 720th second the location of the tunnel is full of smoke, and filled time has a long duration, until 33th minutes the smoke begin to decline, the bottom of the mark bar is visible, when the experiment ends, at this time there is still a small number of smoke at the top of the tunnels.

It can be seen that, if the height of the Ceiling screen in the tunnel is not enough, it can not effectively prevent the spread of smoke to the tunnel, can only play the role of slowing down the speed, but when the height of the wall is high enough, the tunnel will form a closed space, which is not conducive to the personnel escape.

\section{Conclusion}

When there is a fire at one of complex tunnels, this paper makes a comparison about the smoke prevention effect of longitudinal ventilation and ceiling screen of the tunnel which is parallel to it by temperature changes and experimental images. It is concluded that the air curtain is formed at the tunnel intersection by means of longitudinal ventilation, which can effectively block the smoke get into the tunnel, and the flow of air in the tunnel will help to reduce the temperature in the tunnel. In addition, that the tunnel under that mode is still in the connected state helps people escape. When using ceiling screen, if the height of the smoke wall is insufficient, it can only slow down the spread of smoke in the tunnel, can not completely limit the spread of smoke, and will lead to the tunnel temperature gradually increased, which is not conducive to the personnel escape. 


\section{References}

[1] Zheng Z D, Hou L F. Study on the Effect of Automatic Smoke Screen on Tunnel Fire[J]. Safety \& Environmental Engineering, 2011.

[2] Kunsch J P. Simple model for control of fire gases in a ventilated tunnel[J]. Fire Safety Journal, 2002, 37(1):67-81.

[3] Yang Y C, Jia-Peng H E, Zhou R, et al. Study on vertical air jet and hang wall combined smoke control[J]. Fire Science \& Technology, 2011.

[4] WANG Huan, QI Qingjie, JIANG Haiyang, et al. Study on smoke movement characteristics in long - narrow channel with smoke barrier. [J]. China Safety Science Journal, 2017(6):150-156.

[5] Delichatsios M A. Flow of fire gases under a beamed ceiling[J]. Combustion \& Flame, 1981, 43:1-10.

[6] Liu S J,Discussion on application and research of smoke retaining wall[J]. Fire Technique and Products Information, 1996(11):33-36.

[7] Lea J F, Nickens H V, Wells M R. Chapter 3 - Critical velocity[M]// Gas Well Deliquification. Elsevier Inc. 2008.

[8] Ying Z G. Subway station smoke control based on the air curtain and smoke-proof pendant wall[J]. Fire Science \& Technology, 2012.

[9] Sun S X, Zhang Y X,Lei P S, et al , Application conditions of Froude criteria for fire model test of highway tunnel[J]. China Journal of Highway and Transport,2016,29(5):103-108 